
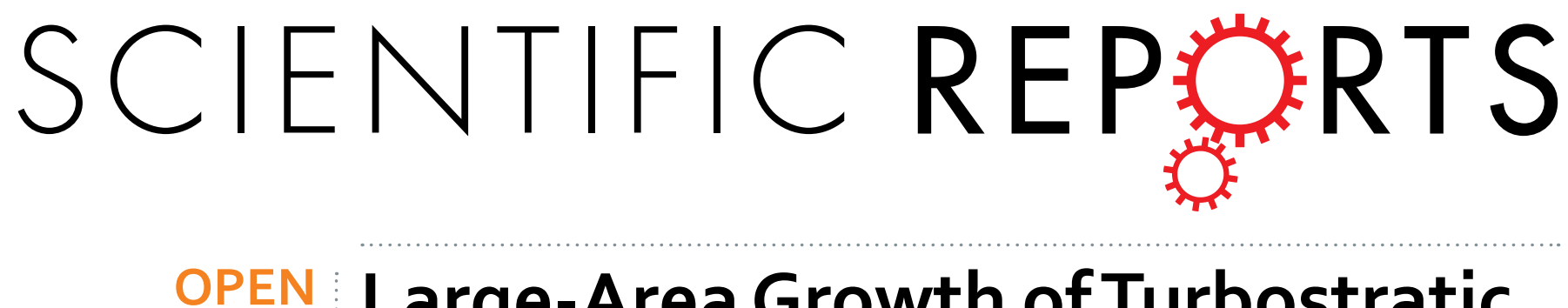

\title{
Large-Area Growth of Turbostratic Graphene on Ni(111) via Physical Vapor Deposition
}

Received: 02 April 2015

Accepted: 07 December 2015

Published: 29 January 2016

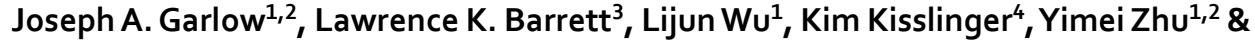
Javier F. Pulecio ${ }^{1}$

Single-layer graphene has demonstrated remarkable electronic properties that are strongly influenced by interfacial bonding and break down for the lowest energy configuration of stacked graphene layers (AB Bernal). Multilayer graphene with relative rotations between carbon layers, known as turbostratic graphene, can effectively decouple the electronic states of adjacent layers, preserving properties similar to that of SLG. While the growth of AB Bernal graphene through chemical vapor deposition has been widely reported, we investigate the growth of turbostratic graphene on heteroepitaxial $\mathrm{Ni}(111)$ thin films utilizing physical vapor deposition. By varying the carbon deposition temperature between $800-1100^{\circ} \mathrm{C}$, we report an increase in the graphene quality concomitant with a transition in the size of uniform thickness graphene, ranging from nanocrystallites to thousands of square microns. Combination Raman modes of as-grown graphene within the frequency range of $1650 \mathrm{~cm}^{-1}$ to $2300 \mathrm{~cm}^{-1}$, along with features of the Raman 2D mode, were employed as signatures of turbostratic graphene. Bilayer and multilayer graphene were directly identified from areas that exhibited Raman characteristics of turbostratic graphene using high-resolution TEM imaging. Raman maps of the pertinent modes reveal large regions of turbostratic graphene on $\mathrm{Ni}(111)$ thin films at a deposition temperature of $1100^{\circ} \mathrm{C}$.

Graphene synthesis originated in the 1960 s with graphitization of $\mathrm{SiC}^{1}$ as well as through chemical vapor deposition (CVD) on $\mathrm{Pt}(111)^{2}$. It was not until Novoselov and Giem established micromechanical cleavage of bulk graphite as a repeatable method to isolate single-layer graphene (SLG) ${ }^{3}$ that graphene began to receive tremendous interest from academia and industry ${ }^{4}$. To date, graphene has been fabricated from a range of carbon sources through a variety of top-down and bottom-up approaches including, mechanical and chemical exfoliation of graphite ${ }^{5}, \mathrm{CVD}^{6,7}$, molecular beam epitaxy ${ }^{8,9}$, graphitization of $\mathrm{SiC}^{10}$, longitudinal unzipping of carbon nanotubes $^{11}$ and, growth from solid carbon sources, such as polymers ${ }^{12}$. Widely considered a prominent growth technique, CVD has produced graphene on the meter scale ${ }^{13}$, and through precise constraint of growth parameters, has demonstrated control over the morphology ${ }^{14}$. Despite these notable advances, out-of-plane $\pi$-orbital hybridization between SLG and its substrate ${ }^{15}$ can limit its theoretical capacity due to charge scattering ${ }^{16}$.

SLG is a two-dimensional network of $\mathrm{sp}^{2}$ bonded carbon atoms that exhibits near-ballistic transport of electrons ${ }^{17}$, among other remarkable properties ${ }^{18}$, enabling a broad range of applications from graphene field effect transistors ${ }^{19}$ for carbon-based electronics to molecular sieves ${ }^{20}$ for water treatment. As a true two-dimensional system, graphene provides an intriguing opportunity to study the fundamental surface physics and interfacial interactions and, in turn, their property/function relations. For example, recent graphene studies have focused on the impact of interfacial interactions on charge transportation ${ }^{17}$ and spin injection efficiency ${ }^{21}$. It is known that interfacial interactions between graphene and its substrate drastically alter the electronic structure of SLG ${ }^{15}$. Moreover, interlayer coupling between adjacent carbon layers in multilayer graphene (MLG) also modifies the dispersion of electronic states and can open a band-gap ${ }^{22}$, suppressing desirable properties of SLG.

Relative rotations between graphene layers could provide a novel route to overcome the restrictions interfacial interactions impose on the desirable electronic properties of SLG. Relative rotations between adjacent graphene

${ }^{1}$ Condensed Matter Physics and Material Science Department, Brookhaven National Laboratory, Upton, NY 11973. ${ }^{2}$ Material Science and Engineering Department, Stony Brook University, Stony Brook, NY 11794. ${ }^{3}$ Division of Materials Science and Engineering, Boston University, Boston, MA 02215. ${ }^{4}$ Center for Functional Nanomaterials, Brookhaven National Laboratory, Upton, NY 11973. Correspondence and requests for materials should be addressed toY.Z. (email: zhu@bnl.gov) or J.F.P. (email: javier.f.pulecio@gmail.com) 
(a)
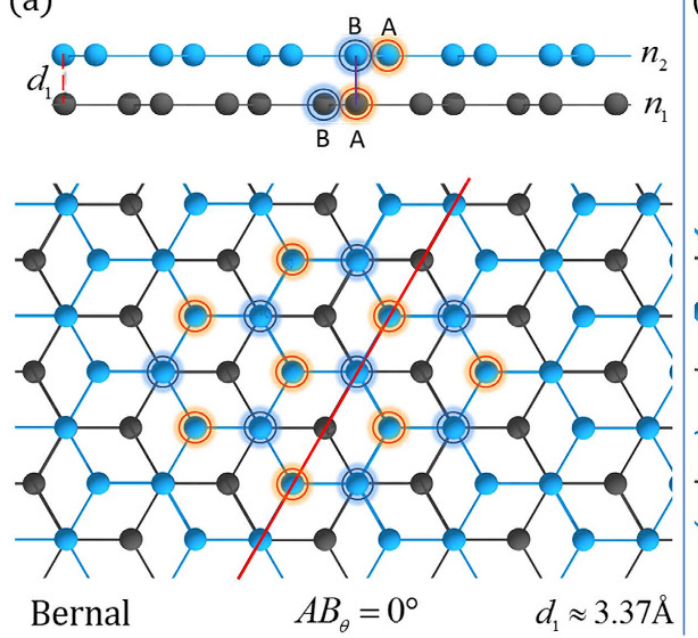

(b)

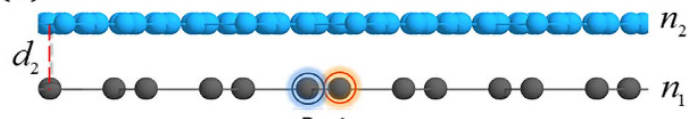

B A

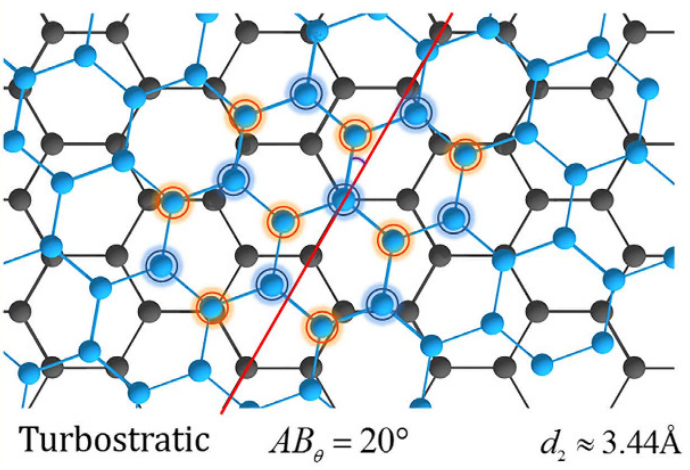

Figure 1. A representative illustration of bilayer graphene with different stacking orientations. (a)Bernal phase, or AB stacked, BLG refers to the stacking of the B site (highlighted navy rings) from the top light blue graphene layer $n_{2}$, directly above the A site (highlighted orange rings) for the bottom grey graphene layer $n_{1}$. This type of stacking has a relative site rotation of $A B=0^{\circ}$ for the neighboring layers $\left(n_{1}, n_{2}\right)$ resulting in orbital hybridization and an interplanar distance of $d_{1}=3.37 \dot{\mathrm{A}}$. (b) Turbostratic graphene is a random relative rotation between adjacent layers, in this case $A B=20^{\circ}$, which mitigates the orbital hybridization and has an increased interplanar distance of $d_{2}=3.44 \dot{\mathrm{A}}$. The coupling between the stacking orientations is different due to the orbital interactions and can be studied using characteristic Raman bands.

layers can alleviate $\pi$-orbital hybridization, thus, restoring the electronic structure of SLG in a MLG configuration $^{23}$. A schematic illustration of layered graphene films with and without relative layer rotations can be seen in Fig. 1. The honeycomb lattice of graphene is composed of two overlapping triangular sublattices, A (orange highlight) and B (blue highlight). For AB Bernal stacked bilayer graphene (BLG), the B site of the top layer sits directly above the A site of the bottom layer leading to electronic coupling between the two layers as shown in the cross-sectional view of Fig. 1a. Figure $1 \mathrm{~b}$ shows a similar bilayer where the top layer is rotated $20^{\circ}$ about the central AB site. Rotational stacking mitigates interlayer coupling, increasing interplanar spacing, and produces unique phonon based Raman spectral signatures ${ }^{24,25}$. In fact, MLG with a relative rotation between layers greater than $3^{\circ}$ maintains linear dispersion of the valence and conduction bands in the low energy regime with a renormalized Fermi velocity, whereas rotations above $20^{\circ}$ effectively decouple layers, preserving the unique electronic states of SLG ${ }^{26}$. Thus, MLG with stacking order that does not subdue the electronic properties of SLG could enable the ballistic transport of electrons through decoupled graphene layers.

Turbostratic graphene is comprised of multiple graphene layers with interlayer rotations that alleviate orbital hybridization resulting in carrier mobilities similar to that of SLG. Its relevance has been limited by the difficulties associated with its controlled and reproducible growth, along with its quantitative characterization. Turbostratic graphene has been grown tens of layers thick on $\mathrm{SiC}$ and observed through Moiré patterns using STM ${ }^{27-29}$, though its integration onto other substrates presents challenges. Additionally, localized areas of twisted bilayer and few-layer graphene have been investigated using characteristic Raman signatures after growth through CVD on $\mathrm{Cu}$ or by manipulating mechanically exfoliated graphene sheets $\mathrm{s}^{30-37}$. While these findings are motivating, the properties of as-grown turbostratic graphene and its possible application remain largely unexplored. Here, heteroepitaxial $\mathrm{Ni}(111)$ thin films were grown on magnesium oxide $(\mathrm{MgO}(111))^{38,39}$ followed by the direct deposition of carbon at substrate temperatures $800^{\circ} \mathrm{C}, 900^{\circ} \mathrm{C}, 1000^{\circ} \mathrm{C}$ and $1100^{\circ} \mathrm{C}$, in a continuous process via physical vapor deposition (PVD) (see Methods section). We systematically investigate the prevalence of as-grown turbostratic graphene with combination Raman modes within the region $1650-2300 \mathrm{~cm}^{-1} 30,31$, various features of the $2 \mathrm{D}$ peak ${ }^{25,40}$ and electron microscopy.

\section{Results and Discussion}

Graphene growth on $\mathrm{Ni(111).} \mathrm{Graphene} \mathrm{has} \mathrm{been} \mathrm{grown} \mathrm{on} \mathrm{a} \mathrm{variety} \mathrm{of} \mathrm{transition} \mathrm{metal} \mathrm{surfaces} \mathrm{such} \mathrm{as}$ $\mathrm{Co}(0001), \mathrm{Cu}(111), \operatorname{Pd}(111), \operatorname{Pt}(111), \operatorname{Ir}(111), \mathrm{Ru}(0001), \mathrm{Ni}(111)$ and polycrystalline nickel. Single crystal substrates are advantageous growth templates as they increase homogeneity of the grown graphene films through a reduction in nucleation sites ${ }^{41,42}$. The $\mathrm{Ni}(111)$ lattice plane is a desirable graphene substrate due to its close lattice match with graphene; the lattice parameter of graphene is $246 \mathrm{pm}$ while $\mathrm{Ni}(111)$ has a lattice parameter of $249 \mathrm{pm}$. The surface morphology of $\mathrm{Ni}(111)$ thin films is comprised of steps and terraces ${ }^{39}$, as shown in Fig. 2a. The growth of graphene on nickel at high temperatures is a dynamic process involving a combination of sub-surface atomic interactions and nucleation mechanisms ${ }^{43,44}$. Carbon diffuses into the heated nickel substrate then graphene nucleates on the nickel surface through precipitation at step-edges, and segregation on terraces. Graphene precipitation refers to a carbon phase separation and occurs out of the carbon-nickel solution at step-edges during cooling $^{43}$, meanwhile, a segregation phase exists where graphene forms on the nickel terraces below the solubility 

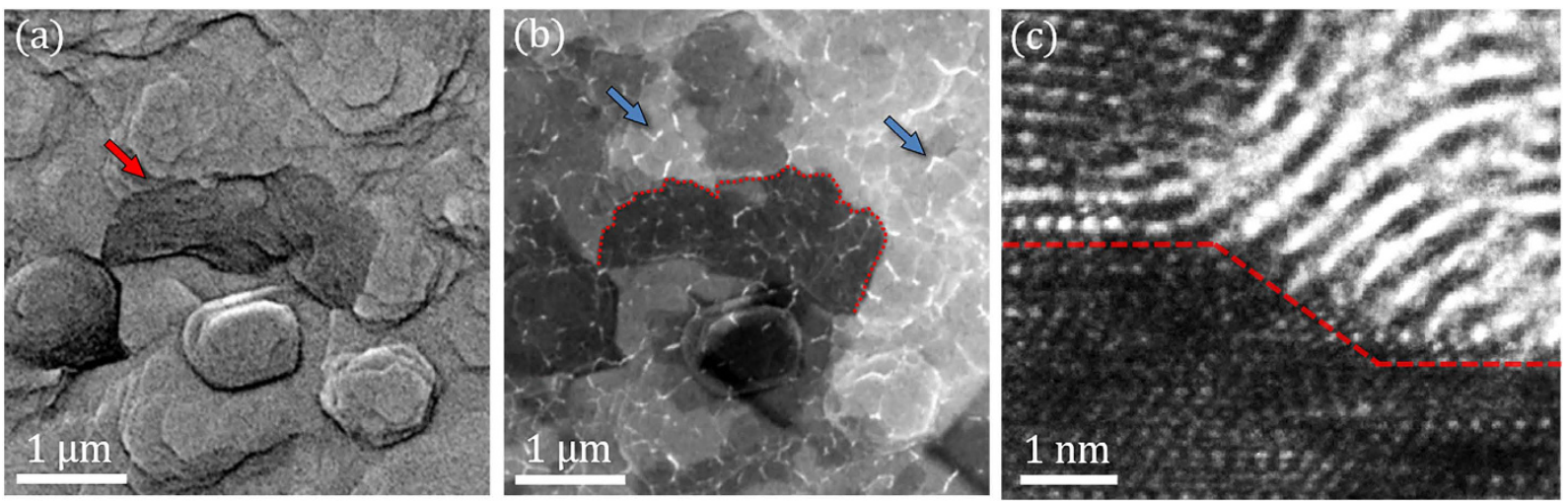

Figure 2. Graphene growth on Ni(111). (a) An SEM image, obtained with an in-lens detector, reveals the surface morphology of the continuous nickel thin film composed of steps and terraces post-graphene growth at $1000^{\circ} \mathrm{C}$. The red arrow shows a prominent step edge. (b) An SEM image of the same region shown in (a), taken with a low angle secondary electron detector that is more sensitive to the graphene layers. The red outline serves to show the same step-edge depicted in (a). The areas of dark contrast correspond to thicker graphene layers due to attenuation of the secondary electrons emitted from the underlying nickel. The nickel thin film is entirely covered with graphene as evidenced by the presence of graphene wrinkles, which appear as bright contrast (blue arrows). (c) A cross-sectional TEM image that exemplifies a graphene growth mechanism on nickel. The red line shows the nickel and graphene interface where the dark region underneath is the nickel substrate. The faceted step edge serves as a nucleation region for thicker multilayer graphene while the higher terrace is covered by bilayer graphene.

limit ${ }^{43}$. Figure $2 \mathrm{~b}$ portrays a graphene patch that nucleated at a nickel step-edge, likely through precipitation during cooling ${ }^{45,46}$. Direct evidence of the relationship between graphene and the nickel step-edge is observed from the TEM image shown in Fig. 2c. It reveals a multilayer graphene domain that nucleated at the nickel step-edge from a cross-sectional perspective and exemplifies a growth mechanism of graphene on nickel. The carbon layers remain bonded to the nickel step-edge, demonstrating their strong interaction. Interestingly, the image shows a bilayer graphene region on top of the terrace (left) which overlays the multilayer domain (right), potentially contributing to the formation of rotationally stacked graphene layers. Graphene precipitation at step-edges is energetically favorable to formation on nickel terraces, inherently generating graphene film heterogeneity due to difference in nucleation rates between the two regions. The deposition temperature and chemical potential critically impact the nucleation rates at the step-edge and at the terrace, modulating graphene film morphology ${ }^{46}$.

Temperature dependent PVD graphene growth. Of recent interest, particularly for deposition on insulating, magnetic and ferroelectric substrates ${ }^{8}$, PVD allows direct deposition onto a desired substrate through sublimation of a graphitic source. PVD at low pressures is an advantageous growth technique that enables line-of-sight deposition with real-time sub-angstrom per second measurement of the atomic carbon flux, in addition to precise control over the quantity of deposited material. Further, it permits direct control over sample temperature and allows the growth of complex heterostructures without breaking vacuum. Specifically for the growth of graphene on carbon-soluble substrates, PVD supports modulation of carbon saturation, the saturation rate, post-deposition annealing and sample cooling rates. Furthermore, it can be tailored to grow graphene directly on a substrate of interest, enabling top-down fabrication techniques to employ as-grown samples in device configurations.

PVD graphene films of varying morphology are presented with representative integrated intensity Raman maps of the graphitic $G$ peak $\left(I_{G}\right)$ in Fig. $3 a-d$, and scanning electron microscopy $(S E M)$ images in Fig. $3 \mathrm{~m}-\mathrm{p}$. Both, analytical techniques, Raman maps and SEM, corroborate an increase in the size of homogenous graphene regions as the deposition temperature is increased. Under the conditions presented here (see methods section), we attribute areas of greater SEM intensity to less graphene layers ${ }^{47}$. For SEM imaging on conducting substrates, electron-beam induced current and differential surface charging do not impact SEM contrast and, thus, contrast is derived directly from secondary electrons, generated by the substrate, attenuated by graphene layers ${ }^{48}$. SEM contrast analysis (not shown here) demonstrates similar layer thicknesses for both the $1000^{\circ} \mathrm{C}$ and $1100^{\circ} \mathrm{C}$ deposition temperatures, although a few marginally thicker regions were sparsely found at the $1000^{\circ} \mathrm{C}$ deposition temperature. At the lower deposition temperatures, $800^{\circ} \mathrm{C}$ and $900^{\circ} \mathrm{C}$, thick graphene patches with lateral dimensions on the order of a few microns were detected. As the deposition temperature increases to $1000^{\circ} \mathrm{C}$ and $1100^{\circ} \mathrm{C}$, regions of constant thickness drastically increase in size with homogenous regions up to $100 \mu \mathrm{m}$ in diameter observed from the $1100^{\circ} \mathrm{C}$ deposition temperature. In conjunction with an increase in the size of distinct graphene regions, histograms of the integrated intensity ratio of the Raman D peak to the G peak (Fig. 3i-l) reveal a dramatic decrease in the quantity of defects relative to the graphitic signal at higher deposition temperatures. For the $1000^{\circ} \mathrm{C}$ and $1100^{\circ} \mathrm{C}$ deposition temperatures, histograms of the $\mathrm{I}_{\mathrm{D}} / \mathrm{I}_{\mathrm{G}}$ ratios (Fig. $3 \mathrm{k}, \mathrm{l}$ ) demonstrate the distribution of relative defects is predominantly less than 0.2 , characteristic of high-quality graphene $e^{49}$. The $\mathrm{I}_{\mathrm{D}} / \mathrm{I}_{\mathrm{G}}$ histograms from the $800^{\circ} \mathrm{C}$ and $900^{\circ} \mathrm{C}$ deposition temperatures show a bell-shaped distribution with mean 


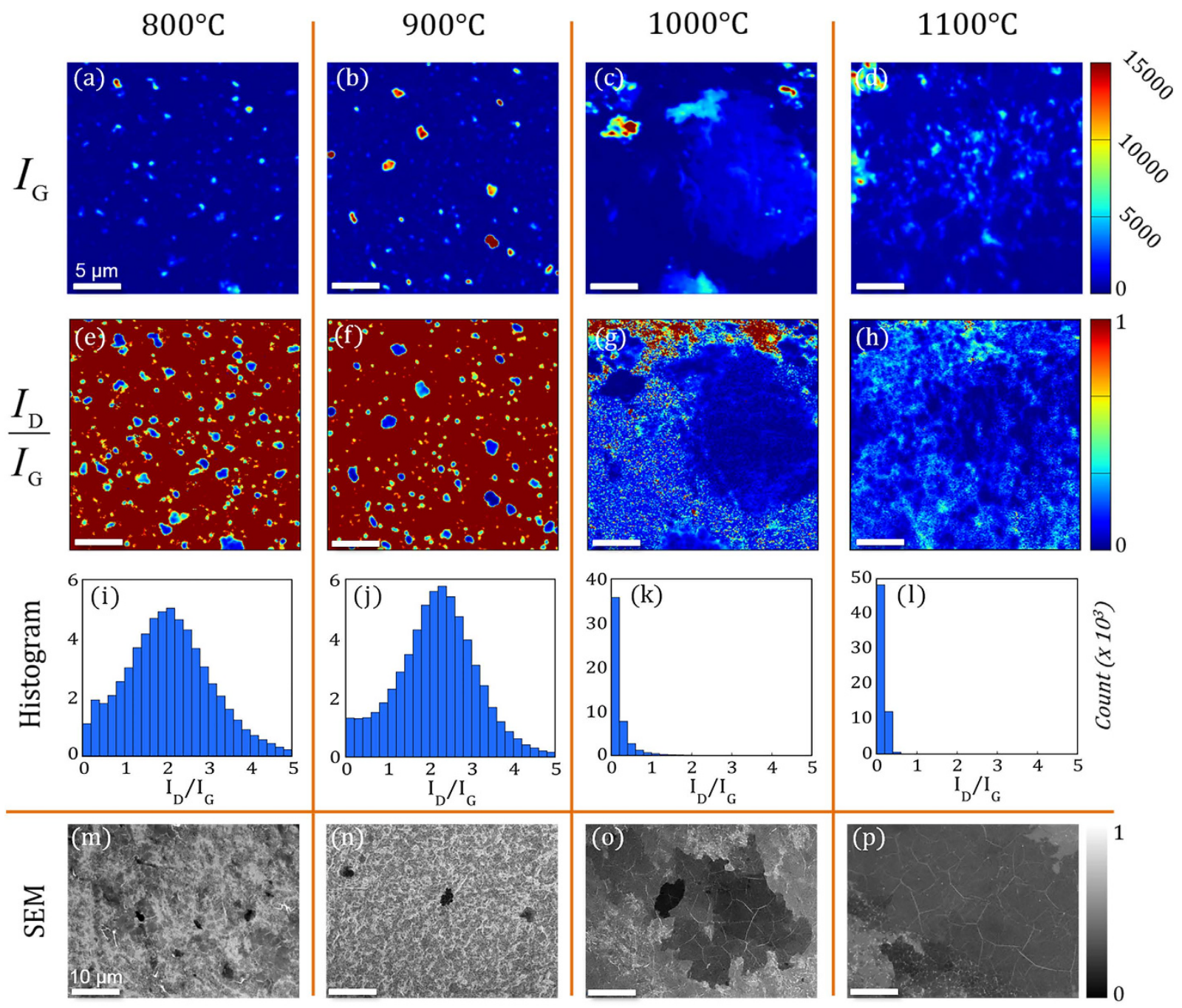

Figure 3. Characterization of the temperature dependent graphene growth morphologies. (a-d) Raman integrated intensity maps of the graphitic, $\mathrm{G}$, phonon mode $\left(\mathrm{I}_{\mathrm{G}}\right)$. Regions with significant $\mathrm{I}_{\mathrm{G}}$ values represent the presence of graphitic growth resolvable by the laser probe. A large graphene patch encompasses the Raman map obtained from the sample deposited at $1100^{\circ} \mathrm{C}$. All Raman maps are obtained from $25 \mu \mathrm{m} \times 25 \mu \mathrm{m}$ areas. $(\mathbf{e}-\mathbf{h})$ Raman integrated intensity ratio maps of the D-peak relative to $G$-peak $\left(\mathrm{I}_{\mathrm{D}} / \mathrm{I}_{\mathrm{G}}\right)$. The $\mathrm{I}_{\mathrm{D}} / \mathrm{I}_{\mathrm{G}}$ maps reveal regions of high quality graphene growth (i.e. a reduction in relative number of defects). (i-l) Histograms of the $\mathrm{I}_{\mathrm{D}} / \mathrm{I}_{\mathrm{G}}$ integrated Raman map raw data quantitate the transition to high quality graphene $(\operatorname{ratios}<0.5)$ at deposition temperatures above $1000^{\circ} \mathrm{C}$. Counts for each $\mathrm{I}_{\mathrm{D}} / \mathrm{I}_{\mathrm{G}}$ bin $\left(0.2\right.$ in size) are three orders of magnitude (Count $\mathrm{x} 10^{3}$ ) higher than the scale bar suggests. (m-p) Representative SEM images of graphene films covering the nickel surface after deposition at each respective deposition temperature. The drastic increase in the size of graphene patches with constant layer thicknesses above $1000^{\circ} \mathrm{C}$ is definitive, and for deposition of $1100^{\circ} \mathrm{C}$, distinct regions up to $100 \mu \mathrm{m}$ in diameter were observed.

values $<2.0$. Using eq. (1), the average nanocrystallite size was calculated as $9.2 \mathrm{~nm}$ and $8.4 \mathrm{~nm}$ at depositions temperatures of $800^{\circ} \mathrm{C}$ and $900^{\circ} \mathrm{C}$, respectively,

$$
L_{a}(\mathrm{~nm})=\left(2.4 \times 10^{-10}\right) \lambda_{l}^{4}\left(\frac{I_{D}}{I_{G}}\right)^{-1}
$$

where $\mathrm{L}_{a}$ is the diameter of the crystallite size, $\lambda_{l}$ is the wavelength of the Raman laser and $\mathrm{I}_{\mathrm{D}} / \mathrm{I}_{\mathrm{G}}$ is the ratio of the integrated intensities of the $D$ and $G$ peak ${ }^{50,51}$. It is apparent, from the $I_{D} / I_{G}$ Raman spectral maps in Fig. 3e,f, that regions of distinct graphene regions are surrounded by nanocrystalline graphene at lower deposition temperatures, while at higher deposition temperatures, large graphene patches are the predominant growth mode. The prominent transition from nanocrystalline graphene to large areas of constant graphene layer thickness, concomitant with a decrease in defects, limits our attention to as-grown PVD graphene deposited at higher temperatures. 

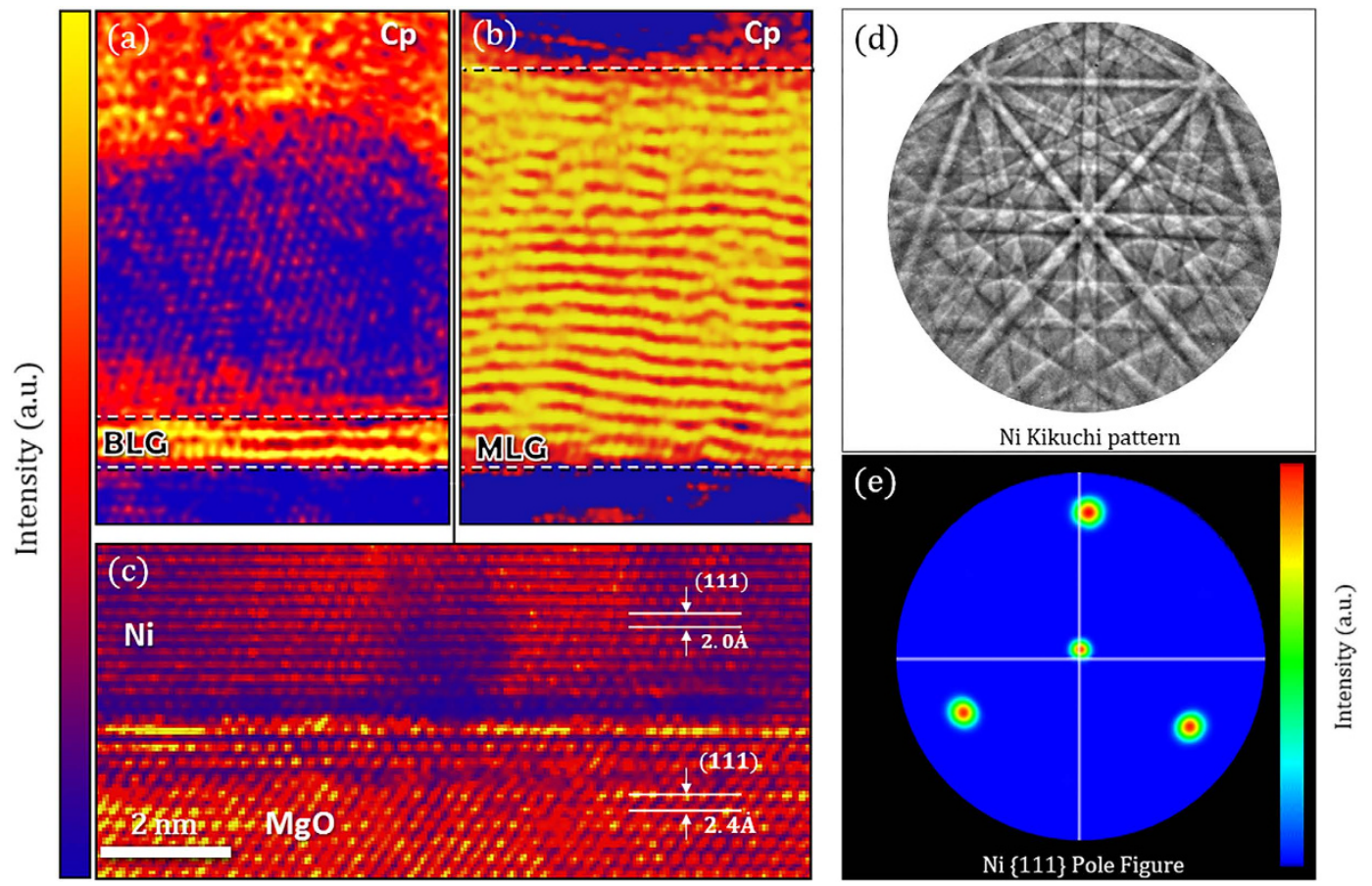

Figure 4. Cross-sectional TEM images of the $\mid$ Magnesium Oxide (MgO) $\mid$ Nickel (Ni) $\mid$ Graphene (Gr) | heterostructure grown at $1100^{\circ} \mathrm{C}$ with a protective capping layer $(\mathrm{Cp})$ used for focused ion beam lift-out (colored to enhance contrast). (a) A representative cross-sectional area where bilayer graphene lattice fringes were observed (yellow). (b) An accompanied cross-sectional TEM image portraying multiple layers of graphene (MLG). BLG and MLG were the only observed growth modes from regions that express turbostratic Raman signatures. (c) The $\mathrm{MgO}$ - nickel substrate on which the graphene layers were grown. The respective distances between the $\mathrm{Ni}$ and $\mathrm{MgO}$ crystal planes were $2.0 \dot{\mathrm{A}}$ and $2.4 \dot{\mathrm{A}}$ as expected for growth along the [111] direction. (d) The Ni Kikuchi pattern obtained from electron back-scattered diffraction (EBSD) demonstrating the high quality crystal growth. (e) An EBSD pole figure taken from a $250 \times 150$ um area showing the $\{111\}$ crystal orientation.

To quantitatively determine the number of graphene layers grown via PVD and investigate the quality of the underlying heteroepitaxial nickel thin film, complementary TEM images were taken. Figure 4a,b displays cross-sectional TEM images from regions of as-grown graphene deposited at $1100^{\circ} \mathrm{C}$. The brighter lattice fringes (yellow) result from an increase in electron beam transmittance (i.e. intensity) due to the lower atomic density of carbon relative to the underlying nickel. Figure 4a identifies BLG while Fig. 4b displays MLG. Figure 4c-e confirms the single-crystalline nature of the nickel thin film is maintained after graphene growth with TEM imaging and electron back-scattered diffraction. From Fig. 4c, the atomic planes of the crystalline nickel mirrors the orientation of the $\mathrm{MgO}$ crystal where the respective $2.0 \dot{\mathrm{A}}$ and $2.4 \dot{\mathrm{A}}$ interplanar distances correspond to the expected (111) crystal planes. Furthermore, electron back-scattered diffraction (EBSD) identifies the Ni crystal orientation over larger areas. Figure 4d shows a representative Kikuchi pattern obtained from the EBSD analysis where the high quality crystal growth is evident by the clear indices and Kikuchi bands found in the $1100^{\circ} \mathrm{C} \mathrm{Ni}$ film (see the methods section for more details). The pole figure shown in Fig. $4 \mathrm{e}$ demonstrates the single $\{111\} \mathrm{Ni}$ crystal orientation taken at steps of $290 \mathrm{~nm}$, probed for a total area of over 5 square mm. Finer maps with steps of $29 \mathrm{~nm}$ were also taken to ensure the microstructure was not varying at smaller scales. Importantly, these cross-sectional areas were obtained from graphene regions exhibiting key turbostratic Raman signatures detailed in subsequent sections and are representative of PVD graphene growth after carbon deposition at $1000^{\circ} \mathrm{C}$ and $1100^{\circ} \mathrm{C}$. The Raman selection rule renders the Raman signal from SLG absent on nickel ${ }^{25,52}$ and SLG was not observed from any of the cross-sectional samples examined. This allows for a detailed analysis of characteristic turbostratic Raman signatures by deconvoluting features that could be attributed to both single-layer and turbostratic graphene as a result of their similar electronic structure.

Characteristic Raman bands for PVD graphene growth. Raman spectroscopy probes the electronic and vibrational properties of molecules and crystalline materials through inelastic scattering of photons by phonons and is a powerful tool for graphene characterization ${ }^{40}$. In brief, the prominent Raman active phonon modes of graphene are known as the D, G and 2D (also known as $\mathrm{G}^{35}$ ) bands. The 2D mode is an overtone of the in-plane transverse optic (iTO) branch and is observed with a Raman Stokes shift of $2700 \mathrm{~cm}^{-1}$. It displays a single Lorentzian profile for SLG, while for AB Bernal MLG, the 2D mode splits into multiple components, broadening its shape as a result of the altered band structure and dispersion near the Fermi level ${ }^{22,53}$. Overall, the presence, position, full width at half maximum (FWHM), integrated intensity $(I)$ and line-shape of Raman spectral features 


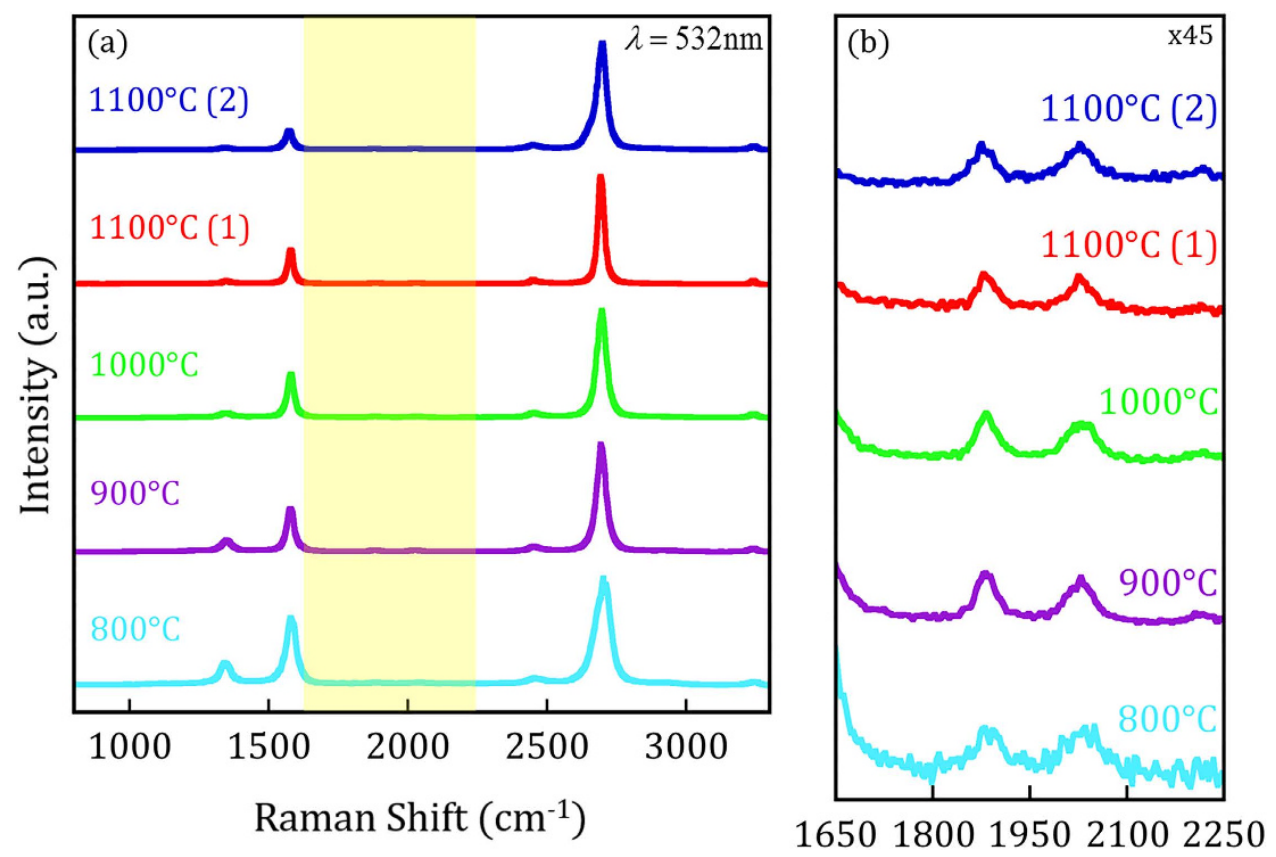

Figure 5. Normalized Raman spectra raw data using a laser excitation energy of $E_{L}=2.33 \mathrm{eV}$, obtained for growth temperatures $800-1100{ }^{\circ} \mathrm{C}$. The spectra taken from the $1100^{\circ} \mathrm{C}$ sample was acquired from the corresponding colored markers shown in Fig. 8a. (a) Raman spectra indicating the high quality graphene growth through PVD. The reduction in D-peak intensity as deposition temperature increases agrees with the $\mathrm{I}_{\mathrm{D}} / \mathrm{I}_{\mathrm{G}}$ Raman maps and SEM images presented in Fig. 3. The area highlighted in yellow signifies the location of combination Raman modes magnified in (b). The peak shape, intensity, and location of the $\mathrm{TS}_{1}$ and $\mathrm{TS}_{2}$ combination Raman modes are indicative of turbostratic stacking.

provide quantitative and qualitative information about the structure and properties of graphene ${ }^{40}$, including the interlayer coupling, which is significant in the context of this study $y^{30,31,35,54-57}$. For more detailed information regarding the characterization of graphene via Raman spectroscopy, we refer the reader to several insightful reviews ${ }^{25,40,49,51,58-60}$.

Figure 5a displays normalized Raman spectra of as-grown PVD graphene from each respective deposition temperature. The data for the samples deposited at $1100^{\circ} \mathrm{C}$ were obtained from the areas indicated with a colored marker in the 2D Raman maps of Fig. 8a. The G peak is primarily a response to an in-plane phonon mode found in graphitic carbon where its intensity with respect to the $2 \mathrm{D}$ peak is often used to qualify the number of $A B$ stacked graphene layers ${ }^{53}$. The $\mathrm{D}$ peak is active in the presence of structural defects or graphene edges. The normalized Raman spectra presented in Fig. 5 demonstrate the high-quality growth of graphene on nickel through PVD and, in agreement with Fig. 3, exhibit a reduction in the relative D-peak intensity as deposition temperature increases. Specific values for the spectra in Fig. 5 have been provided in Table S1 for reference. A detailed examination of the $2 \mathrm{D}$ peak features can provide further details of the graphene growth due to its sensitivity to the electronic and phonon band structure ${ }^{40,59}$.

The normalized Raman spectra, shown in Fig. 5a, reveal a 2D peak intensity significantly greater than the G peak intensity for PVD deposition temperatures above $800^{\circ} \mathrm{C}$, consistent with turbostratic graphene. The profile of the 2D phonon mode of turbostratic graphene portrays that of SLG and, therefore, is composed of a single Lorentz peak resulting from the linear band dispersion of the weakly coupled layers ${ }^{24,25,32,40,61}$. Turbostratic graphene has an active Raman signal on nickel ${ }^{25}$, despite its similar electronic structure to SLG. The 2D FWHM of turbostratic graphene is distinct from that of ordered AB Bernal MLG, which successively broadens as layer numbers increase ${ }^{40,53,59}$. The $2 \mathrm{D}$ peak, from samples deposited above $800^{\circ} \mathrm{C}$, fit well to a single Lorentzian and their observed FWHM are narrower than would be expected for AB MLG. Figure 6 presents the 2D peak FWHM from the spectra presented in Fig. 5a along with the reported 2D FWHM for rotated graphene bilayers by Kim et al. ${ }^{32}$. The 2D FWHM from all samples presented in Fig. 5a fall within the range of the reported rotated graphene bilayers with a $2 \mathrm{D} \mathrm{FWHM}$ as low as $27 \mathrm{~cm}^{-1}$, observed from the $1100^{\circ} \mathrm{C}(1)$ spectra. A $2 \mathrm{D} \mathrm{FWHM}$ of this magnitude is on the order of the 2D FWHM reported for $\mathrm{SLG}^{53}$ as well as for twisted BLG with high angle layer rotations ${ }^{32,35}$. Interestingly, the plot suggests the relative rotation between graphene layers increases as deposition temperature increases.

Combination Raman Modes. Rao et al..$^{30}$ and Cong et al. ${ }^{31}$ have reported combination Raman modes between $1650 \mathrm{~cm}^{-1}$ and $2300 \mathrm{~cm}^{-1}$ differ in intensity and position for SLG, turbostratic (i.e. incommensurate) and Bernal phase (i.e. commensurate) MLG. Of particular interest are the $\mathrm{iTALO}^{-}$, $\mathrm{iTALO}^{+}$, iTOLA, and LOLA modes that are distinct for turbostratic graphene and become convoluted for AB Bernal stacked graphene layers. The $\mathrm{iTALO}^{-}, \mathrm{iTALO}^{+}$, iTOLA and LOLA modes are believed to arise from the combination of in-plane transverse 


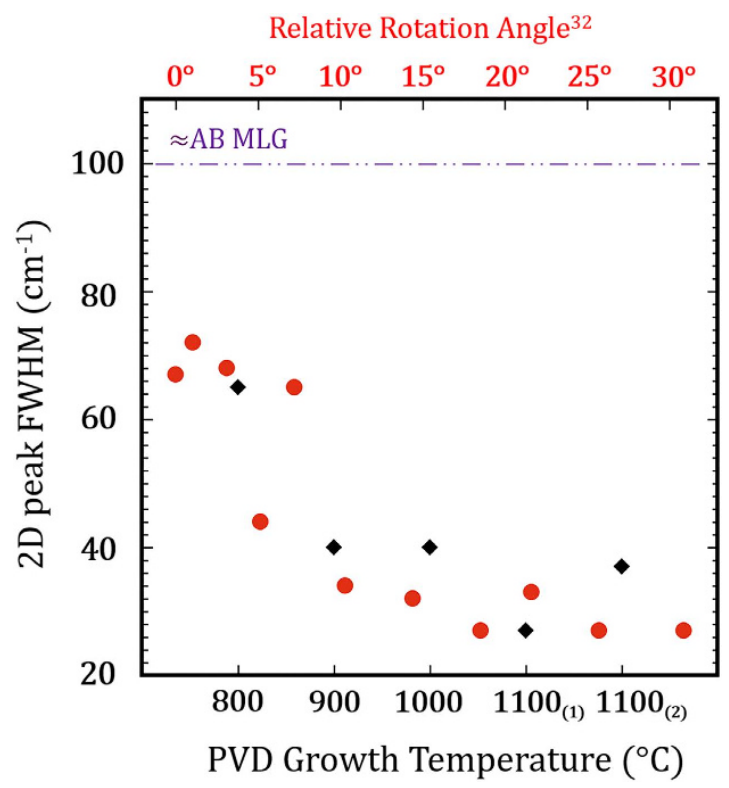

Figure 6. The 2D full width at half maximum (black markers) of the Raman spectra presented in Fig. 5 as a function of deposition temperature. The red markers were adapted from Kim et al. ${ }^{32}$ showing the 2D FHWM as a function of the rotation angle for twisted BLG. The 2D FWHM for turbostratic PVD graphene trends downward towards the expected values for SLG as the deposition temperature increases. At high deposition temperatures, 2D FWHM values as low as $27 \mathrm{~cm}^{-1}$ may suggest high relative rotation angles. The purple dashed line indicates the expected 2D FWHM for Bernal stacked graphene layers.

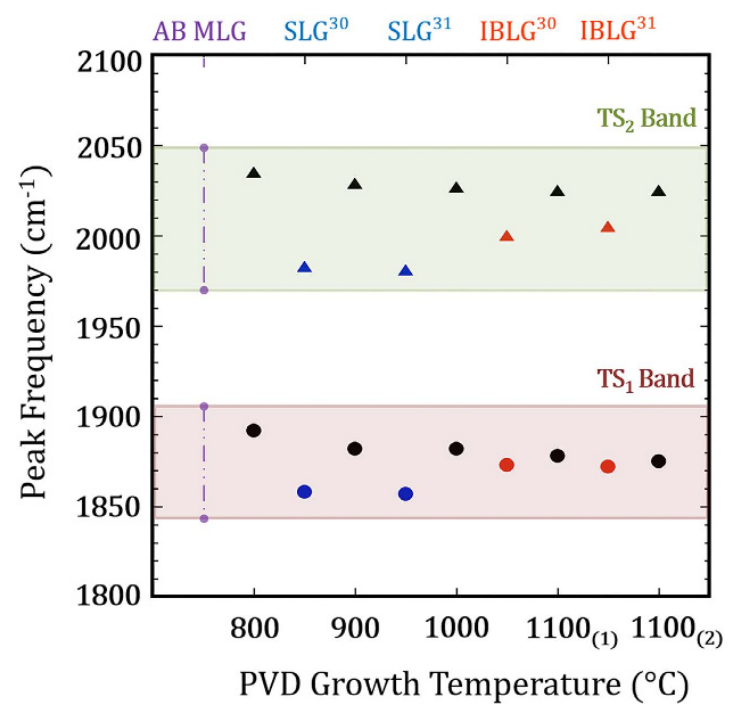

Figure 7. Combination Raman modes of turbostratic graphene compared to reported literature values. The $\mathrm{TS}_{1}$ (circles) \& $\mathrm{TS}_{2}$ (triangles) peak positions for different PVD deposition temperatures, shown with black markers. The SLG (blue) and IBLG (red) where added from Rao et al..$^{30}$ and Cong et al. ${ }^{31}$ for reference. The peak positions of $\mathrm{TS}_{1}$ and $\mathrm{TS}_{2}$ are blue-shifted from the SLG and IBLG values. At $1100^{\circ} \mathrm{C}$ growth temperatures, the $\mathrm{TS}_{1}$ peak nearly matches the position observed from IBLG while the $\mathrm{TS}_{2}$ peak remains blue-shifted from IBLG peak positions. The purple vertical dashed line represents the absence of prominent peaks for AB Bernal MLG.

acoustic (iTA) and the longitudinal optic (LO), iTA and longitudinal acoustic (LA) and LO + LA modes. Here, we designate the iTALO $^{-}$mode as TS $_{1}$ and the iTOLA/LOLA modes as TS 2 . Additionally, the iTOTA, R and R' Raman phonon modes, can also be used to classify interlayer coupling ${ }^{30}$ and the relative layer rotation angle between graphene layers ${ }^{33-35,62}$. While these peaks were detected within turbostratic regions of our samples, their presence was inconsistent.

Figure $5 \mathrm{~b}$ magnifies the frequency range where combination Raman modes reside and presents the same spectra shown in Fig. 5a. For turbostratic graphene, the $\mathrm{TS}_{1}$ and $\mathrm{TS}_{2}$ modes are well defined with distinct intensities 

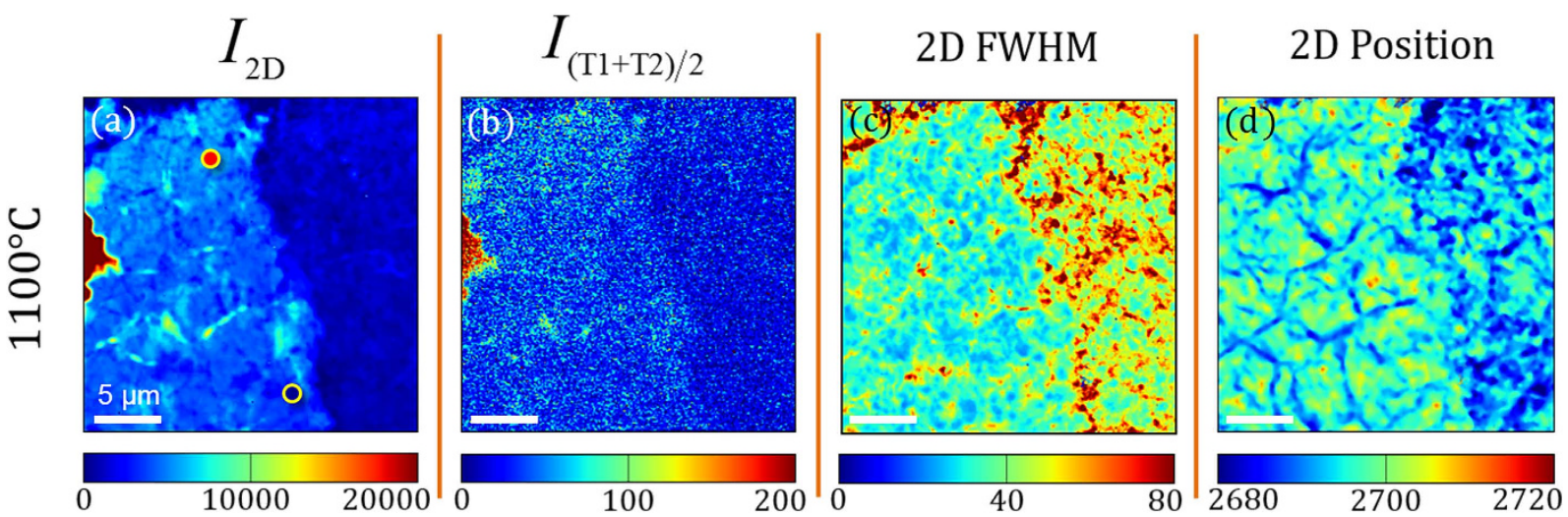

Figure 8. Characteristic Raman maps demonstrating the prevalence of turbostratic graphene over a $25 \mu \mathrm{m} \times 25 \mu \mathrm{m}$ area from a sample deposited at $1100^{\circ} \mathrm{C}$. The figure displays Raman maps of the (a) integrated 2D peak intensity, (b) average integrated intensity of the TS peaks, (c) 2D full width at half maximum (FWHM) and (d) frequency of the 2D peak. The region of increased 2D integrated intensity, located on the left-half of the figure, spatially corresponds with the presence of combination Raman modes, narrower 2D peak FWHM and an upshift in 2D peak frequency. Color markers present in Fig. 8a indicate where spectra of the same line colors were acquired in Fig. 5.

while these peaks disperse into the background for AB Bernal BLG and MLG. Further, turbostratic graphene exhibits greater $\mathrm{TS}_{1}$ and $\mathrm{TS}_{2}$ peak intensities than SLG, due to stiffening of the phonon modes and, importantly, their peak positions are blue-shifted ${ }^{30,31}$. The $\mathrm{TS}_{1}$ and $\mathrm{TS}_{2}$ peaks are clearly observed in Fig. $5 \mathrm{~b}$ with distinct intensity from as-grown samples deposited at $900^{\circ} \mathrm{C}, 1000^{\circ} \mathrm{C}$ and $1100^{\circ} \mathrm{C}$. The $\mathrm{TS}_{1}$ (circles) and $\mathrm{TS}_{2}$ (triangles) peak positions are displayed for all deposition temperatures in Fig. 7. To serve as a reference, the reported TS peak positions for SLG (blue markers) and incommensurate BLG (IBLG) (red markers) have also been added from Rao et al..$^{30}$ and Cong et al. ${ }^{31}$. The purple line in Fig. 7, labeled AB MLG, is used to represent the absence of clear $\mathrm{TS}$ peaks from $\mathrm{AB}$ Bernal graphene layers. The $\mathrm{TS}_{1}$ and $\mathrm{TS}_{2}$ peak frequencies are blue-shifted for all deposition temperatures and the magnitude of their blue-shift is reduced as deposition temperature is increased (Fig. 7). Moreover, the turbostratic graphene $\mathrm{TS}_{1}$ peak frequencies shown here closely resemble the reported $\mathrm{TS}_{1}$ peak positions of IBLG (i.e. turbostratic BLG) while the turbostratic $\mathrm{TS}_{2}$ peaks are upshifted from reported $\mathrm{IBLG}_{\mathrm{TS}}$ peak frequencies. This observation is clear when examining the $1100^{\circ} \mathrm{C}$ samples; the $\mathrm{TS}_{1}$ peak position is within $2 \mathrm{~cm}^{-1}$ of the reported IBLG values while the $\mathrm{TS}_{2}$ peak position is found at higher frequencies. The increased blue-shift seen in the $\mathrm{TS}_{2}$ peak is consistent across all deposition temperatures and could result from an intrinsic difference between the phonon modes of IBLG and as-grown turbostratic graphene investigated here. Finally, the $\mathrm{M}$ band $\mathrm{d}^{30,31}$, which is an overtone of the oTO mode within the wavelength $1650-1750 \mathrm{~cm}^{-1}$ that is present for Bernal phase BLG and MLG, was not detected in our PVD graphene, further supporting the layer rotations conclusion. The presence of as-grown PVD turbostratic graphene is systematically established with features of the 2D peak, combination Raman modes, in conjunction with high-resolution TEM analysis, our focus proceeds to mapping turbostratic graphene with pertinent Raman signatures.

Mapping Turbostratic Graphene. The Raman maps of the integrated intensity of the 2D peak $\left(\mathrm{I}_{2 \mathrm{D}}\right)$, averaged TS peaks $\left(\mathrm{I}_{\left(\mathrm{TS}_{1}+\mathrm{TS}_{2}\right) / 2}\right), 2 \mathrm{D}$ FWHM and $2 \mathrm{D}$ peak frequency, shown in Fig. 8, outline the prevalence of turbostratic graphene on $\mathrm{Ni}(111)$ deposited at $1100^{\circ} \mathrm{C}$. High $2 \mathrm{D}$ integrated intensity values suggest the absence of $\mathrm{AB}$ Bernal stacking, supporting the presence of MLG with properties similar to SLG. The $\mathrm{I}_{2 \mathrm{D}}$ Raman map (Fig. 8a) shows a clear transition in 2D intensity from left $(I>5000)$ to right $(I<5000)$. Traditionally, higher 2D intensities would be associated to $S_{L G}{ }^{53}$, but as explained in the previous section, we attribute the increased intensity to the decoupled nature of turbostratic graphene. A 2D FWHM (Fig. 8c) on the order of $30 \mathrm{~cm}^{-1}$ is observed over the area of interest, indicating this region preserves the electronic structure of $\mathrm{SLG}^{26,61}$. In addition, upshifted $2 \mathrm{D}$ peak frequencies (Fig. 8d), indicative of layer rotations ${ }^{32,59,63,64}$ found in turbostratic graphene, follow the intensity profile of the $\mathrm{I}_{2 \mathrm{D}}$ and 2D FWHM Raman maps which are direct evidence of single Lorentzian Raman peaks and turbostratic graphene $e^{25,40}$. Intriguingly, the Raman map of the $\mathrm{I}_{\left(\mathrm{TS}_{1}+\mathrm{TS}_{2}\right) / 2}$ intensity (Fig. 8b) closely mirrors the $\mathrm{I}_{2 \mathrm{D}}, 2 \mathrm{D}$ FWHM and $2 \mathrm{D}$ peak position profiles. These observations establish the TS peaks as signatures that can be used to spatially map graphene with incommensurate stacking, despite their limited intensity. The regions that exhibit high $\mathrm{I}_{2 \mathrm{D}}, \mathrm{I}_{\left(\mathrm{TS}_{1}+\mathrm{TS}_{2}\right) / 2}$ values, along with narrow $2 \mathrm{D}$ peak widths and an upshifted peak frequency, comprise a substantial area of the $25 \mu \mathrm{m} \times 25 \mu \mathrm{m}$ Raman maps in Fig. 8 and depict domain-like growth of turbostratic graphene on $\mathrm{Ni}(111)$. These Raman maps demonstrate the large-area coverage of turbostratic graphene after carbon deposition at $1100^{\circ} \mathrm{C}$ and exemplify the inherent differences between graphene growth on nickel and graphene grown on copper, where the prevalence of turbostratic graphene is sparse ${ }^{36}$. The spatial identification of large-area turbostratic graphene grown via PVD demonstrated here could be ideal for device architectures that would leverage the properties of SLG in a multi-layered structure. 


\section{Conclusion}

Using physical vapor deposition (PVD), large-area turbostratic graphene was grown on heteroepitaxial $\mathrm{Ni}(111)$ thin films in a continual process with precise control of the deposition temperature, beam flux, and total amount of carbon deposited. SEM images and Raman maps reveal a substantial increase in graphene regions of constant thickness at higher deposition temperatures. Distinct graphene regions of up to $100 \mu \mathrm{m}$ in diameter were observed at carbon deposition temperatures of $1100^{\circ} \mathrm{C}$. Correspondingly, low $\mathrm{I}_{\mathrm{D}} / \mathrm{I}_{\mathrm{G}}$ ratios observed from as-grown samples deposited at $1000^{\circ} \mathrm{C}$ and $1100^{\circ} \mathrm{C}$ represent the high-quality nature of the graphene grown via PVD. High-resolution TEM imaging of focused ion beam prepared cross-sections directly identify bilayer and multilayer graphene (MLG) growth regimes in areas that exhibited characteristic turbostratic graphene Raman signatures. The characteristics of the $2 \mathrm{D}$ Raman mode and combination iTALO ${ }^{-} / \mathrm{iTALO}^{+}\left(\mathrm{TS}_{1}\right)$ and iTOLA/ LOLA $\left(\mathrm{TS}_{2}\right)$ Raman modes were employed to investigate interlayer coupling between as-grown MLG layers. Turbostratic graphene was identified using distinct peak intensities and blue-shifted frequencies of combination Raman modes accompanied by characteristic single Lorentzian 2D peak profiles and direct layer quantification through TEM analysis. Raman maps of turbostratic graphene Raman modes reveal significant coverage of turbostratic graphene on the $\mathrm{Ni}(111)$ at high deposition temperatures using PVD. The spatial identification of large-area turbostratic graphene using PVD on nickel thin films provides a unique opportunity to explore the fundamental properties and viability of turbostratic graphene for future applications such as data storage and transmission technologies.

\section{Experimental Methods}

Sample Preparation. The growth method present here has been adapted from Iwasaki et al. ${ }^{39}$ Heteroepitaxial nickel(Ni) thin films were grown via high-vacuum electron-beam evaporation at base pressures of $2 \times 10^{-9}$ Torr from a $99.999 \% \mathrm{Ni}$ source on single crystalline magnesium oxide $(\mathrm{MgO}(111))$ substrates. Due to the difference in lattice parameters between $\mathrm{Ni}(111)$ and $\mathrm{MgO}(111), 100 \mathrm{~nm}$ of nickel was deposited at $300^{\circ} \mathrm{C}$ to promote single domain growth and decrease the surface roughness of the thin film ${ }^{38}$. Subsequently, an additional $100 \mathrm{~nm}$ of nickel was deposited at $600^{\circ} \mathrm{C}$ to increase the nickel grain size ${ }^{39}$. Following growth of the $\mathrm{Ni}(111)$ substrates, carbon was deposited at a rate of less than $0.5 \AA / \mathrm{s}$ through sublimation of a highly oriented pyrolytic graphite source at sample deposition temperatures of $800^{\circ} \mathrm{C}, 900^{\circ} \mathrm{C} 1000^{\circ} \mathrm{C}$, and $1100^{\circ} \mathrm{C}$. We note, nickel thin films remained continuous with no agglomeration for all deposition temperatures. At high temperature, dissolved carbon segregates on the nickel surface below the solubility limit then readily nucleates once saturation is reached - either through precipitation or as the result of adatoms impinging on the surface ${ }^{43}$. Carbon segregation and precipitation curves for $200 \mathrm{~nm}$ thick Ni thin films can be found in Figure S1. After carbon deposition, samples were cooled to $550{ }^{\circ} \mathrm{C}$ and maintained at this temperature for 2 hours to heal the nickel film ${ }^{17}$.

Raman, SEM, and TEM Characterization. The Raman spectra and Raman maps were taken using a Witec Confocal Raman Microscope Alpha 300R using a $532 \mathrm{~nm}$ laser wavelength. The $25 \mu \mathrm{m} \times 25 \mu \mathrm{m}$ Raman maps were constructed from $250 \times 250$ point spectra. The Raman maps for each deposition temperature were taken of the same region, respectively. For all integrated intensity maps, the $2 \mathrm{D}$ band was designated 2670$2730 \mathrm{~cm}^{-1}$, the $\mathrm{G}$ band was designated $1560-1600 \mathrm{~cm}^{-1}$, and the D band was designated $1325-1375 \mathrm{~cm}^{-1}$. The $\mathrm{TS}_{1}$ and $\mathrm{TS}_{2}$ peaks were designated $1860-1910 \mathrm{~cm}^{-1}$ and $2000-2050 \mathrm{~cm}^{-1}$, respectively. The peak intensity ratios, seen in Table S1, were calculated using the peak height after background subtraction. The bin size for all histograms is 0.2 .

The SEM images were taken using a JEOL 7600F Analytical SEM at an acceleration voltage of $2 \mathrm{kV}$ and a working distance of $3 \mathrm{~mm}$ in order to excite secondary electrons from the graphene layers. The TEM images were taken using a double aberration corrected JEOL ARM 200F at an acceleration voltage of $80 \mathrm{kV}$ to mitigate any possible beam damage. The EBSD data was taken at an acceleration voltage of $20 \mathrm{kV}$ and incidence angle of $70^{\circ}$. The mapping of the crystalline orientation takes into account this $20^{\circ}$ offset geometry from normal which is why the [111] index is just above the pattern shown in Fig. $4 \mathrm{~d}$.

\section{References}

1. Badami, D. V. Graphitization of $\alpha$-Silicon Carbide. Nature 193, 569-570 (1962).

2. May, J. W. Platinum surface LEED rings. Surface Science 17, 267-270 (1969).

3. Novoselov, K. S. et al. Electric field effect in atomically thin carbon films. Science 306, 666-669 (2004).

4. Geim, A. K. \& Novoselov, K. S. The rise of graphene. Nat. Mater. 6, 183-191 (2007).

5. Park, S. \& Ruoff, R. S. Chemical methods for the production of graphenes. Nat. Nanotechnol. 4, 217-224 (2009).

6. Li, X. et al. Large-area synthesis of high-quality and uniform graphene films on copper foils. Science 324, 1312-1314 (2009).

7. Reina, A. et al. Large area, few-layer graphene films on arbitrary substrates by chemical vapor deposition. Nano Lett. 9, 30-35 (2009).

8. Zhou, M. et al. Direct graphene growth on Co3O4(111) by molecular beam epitaxy. J. Phys. Condens. Matter 24, 072201 (2012).

9. Park, J. et al. Epitaxial graphene growth by carbon molecular beam epitaxy (CMBE). Adv. Mater. 22, 4140-4145 (2010).

10. Emtsev, K. V. et al. Towards wafer-size graphene layers by atmospheric pressure graphitization of silicon carbide. Nat. Mater. 8, 203-207 (2009).

11. Kosynkin, D. V. et al. Longitudinal unzipping of carbon nanotubes to form graphene nanoribbons. Nature 458, 872-876 (2009).

12. Sun, Z. et al. Growth of graphene from solid carbon sources. Nature 468, 549-552 (2010).

13. Bae, S. et al. Roll-to-roll production of 30-inch graphene films for transparent electrodes. Nat. Nanotechnol. 5, 574-578 (2010).

14. Mattevi, C., Kim, H. \& Chhowalla, M. A review of chemical vapour deposition of graphene on copper. J. Mater. Chem. 21, 3324 (2011).

15. Fratini, S. \& Guinea, F. Substrate-limited electron dynamics in graphene. Phys. Rev. B 77, 195415 (2008).

16. Chen, J.-H. et al. Charged-impurity scattering in graphene. Nature Physics 4, 377-381 (2008).

17. Du, X., Skachko, I., Barker, A. \& Andrei, E. Y. Approaching ballistic transport in suspended graphene. Nature nanotechnology 3, 491-495 (2008)

18. Zhu, Y. et al. Graphene and graphene oxide: synthesis, properties, and applications. Adv. Mater. 22, 3906-3924 (2010). 
19. Meric, I. et al. Current saturation in zero-bandgap, top-gated graphene field-effect transistors. Nat. Nanotechnol. 3, 654-659 (2008).

20. Joshi, R. K. et al. Precise and ultrafast molecular sieving through graphene oxide membranes. Science 343, 752-754 (2014).

21. Van't Erve, O. M. J.et al. Low-resistance spin injection into silicon using graphene tunnel barriers. Nat. Nanotechnol. 7, 737-742 (2012).

22. Mak, K. F., Sfeir, M. Y., Misewich, J. a. \& Heinz, T. F. The evolution of electronic structure in few-layer graphene revealed by optical spectroscopy. Proc. Natl. Acad. Sci. USA. 107, 14999-15004 (2010).

23. Lopes dos Santos, J. M. B., Peres, N. M. R. \& Castro Neto, A. H. Graphene Bilayer with a Twist: Electronic Structure. Phys. Rev. Lett. 99, 256802 (2007).

24. Latil, S., Meunier, V. \& Henrard, L. Massless fermions in multilayer graphitic systems with misoriented layers: Ab initio calculations and experimental fingerprints. Phys. Rev. B 76, 201402 (2007).

25. Malard, L. M., Pimenta, M. A., Dresselhaus, G. \& Dresselhaus, M. S. Raman spectroscopy in graphene. Phys. Rep. 473, 51-87 (2009).

26. Luican, A. et al. Single-layer behavior and its breakdown in twisted graphene layers. Phys. Rev. Lett. 106, 126802 (2011).

27. Hass, J. et al. Why multilayer graphene on $4 \mathrm{H}-\mathrm{SiC}(000-1)$ behaves like a single sheet of graphene. Phys. Rev. Lett. 100, 125504 (2008).

28. Varchon, F., Mallet, P., Magaud, L. \& Veuillen, J.-Y. Rotational disorder in few-layer graphene films on 6H-SiC(000-1): A scanning tunneling microscopy study. Phys. Rev. B 77, 165415 (2008).

29. Hass, J., de Heer, W. A. \& Conrad, E. H. The growth and morphology of epitaxial multilayer graphene. Journal of Physics: Condensed Matter 20, 323202 (2008).

30. Rao, R., Podila, R., Tsuchikawa, R. \& Katoch, J. Effects of Layer Stacking on the Combination Raman Modes in Graphene. ACS Nano 5, 1594-1599 (2011)

31. Cong, C., Yu, T., Saito, R., Dresselhaus, G. F. \& Dresselhaus, M. S. Second-order overtone and combination Raman modes of graphene layers in the range of 1690-2150 cm-1. ACS Nano 5, 1600-1605 (2011).

32. Kim, K. et al. Raman Spectroscopy Study of Rotated Double-Layer Graphene: Misorientation-Angle Dependence of Electronic Structure. Phys. Rev. Lett. 108, 246103 (2012).

33. Lu, C. et al. Twisting bilayer graphene superlattices. ACS Nano 7, 2587-2594 (2013).

34. Wu, J.-B. et al. Resonant Raman spectroscopy of twisted multilayer graphene. Nat. Commun. 5, 5309 (2014).

35. Carozo, V. et al. Resonance effects on the Raman spectra of graphene superlattices. Phys. Rev. B 88, 085401 (2013).

36. Fang, W. et al. Rapid identification of stacking orientation in isotopically labeled chemical-vapor grown bilayer graphene by Raman spectroscopy. Nano Lett. 13, 1541-1548 (2013).

37. Hwang, J-S. et al. Imaging layer number and stacking order through formulating Raman fingerprints obtained from hexagonal single crystals of few layer graphene. Nanotechnology 24, 015702 (2013).

38. Sandström, P., Svedberg, E. B., Birch, J. \& Sundgren, J.-E. Structure and surface morphology of epitaxial Ni films grown on $\mathrm{MgO}(111)$ substrates: growth of high quality single domain films. J. Cryst. Growth 197, 849-857 (1999).

39. Iwasaki, T. et al. Long-range ordered single-crystal graphene on high-quality heteroepitaxial Ni thin films grown on $\mathrm{MgO}(111)$. Nano Lett. 11, 79-84 (2011).

40. Ferrari, A. C. \& Basko, D. M. Raman spectroscopy as a versatile tool for studying the properties of graphene. Nat. Nanotechnol. 8, 235-46 (2013).

41. Reina, A. et al. Growth of large-area single- and Bi-layer graphene by controlled carbon precipitation on polycrystalline Ni surfaces. Nano Res. 2, 509-516 (2009).

42. Zhang, Y. et al. Comparison of Graphene Growth on Single-Crystalline and Polycrystalline Ni by Chemical Vapor Deposition. J. Phys. Chem. Lett. 1,3101-3107 (2010).

43. Baraton, L. et al. On the mechanisms of precipitation of graphene on nickel thin films. Europhys. Lett. 96, 46003 (2011).

44. Li, H.-B., Page, A. J., Wang, Y., Irle, S. \& Morokuma, K. Sub-surface nucleation of graphene precursors near a Ni(111) step-edge. Chem. Commun. (Camb). 48, 7937-7939 (2012).

45. Li, H.-B., Page, A. J., Wang, Y., Irle, S. \& Morokuma, K. Sub-surface nucleation of graphene precursors near a Ni(111) step-edge. Chem. Commun. 48, 7937-7939 (2012).

46. Gao, J., Yip, J., Zhao, J., Yakobson, B. I. \& Ding, F. Graphene nucleation on transition metal surface: structure transformation and role of the metal step edge. J. Am. Chem. Soc. 133, 5009-5015 (2011).

47. Goldstein, J. et al. Scanning Electron Microscopy and X-ray Microanalysis. (Springer, 2003).

48. Cazaux, J. Material contrast in SEM: Fermi energy and work function effects. Ultramicroscopy 110, 242-253 (2010).

49. Ni, Z., Wang, Y., Yu, T. \& Shen, Z. Raman spectroscopy and imaging of graphene. Nano Res. 1, 273-291 (2010).

50. Cancado, L. G. et al. General equation for the determination of the crystallite size L[sub a] of nanographite by Raman spectroscopy. Appl. Phys. Lett. 88, 163106 (2006).

51. Pimenta, M. A. et al. Studying disorder in graphite-based systems by Raman spectroscopy. Phys. Chem. Chem. Phys. 9, 1276-1291 (2007).

52. Dahal, A. \& Batzill, M. Graphene-nickel interfaces: a review. Nanoscale 6, 2548-2562 (2014)

53. Ferrari, A. C. et al. Raman spectrum of graphene and graphene layers. Phys. Rev. Lett. 97, 187401 (2006).

54. Tan, P. H. et al. The shear mode of multilayer graphene. Nature Materials 11, 294-300 (2012).

55. Lui, C. H. \& Heinz, T. F. Measurement of layer breathing mode vibrations in few-layer graphene. Phys. Rev. B 87, 121404 (2013).

56. Sato, K. et al. Raman spectra of out-of-plane phonons in bilayer graphene. Phys. Rev. B 84, 035419 (2011).

57. Cong, C. et al. Raman characterization of ABA- and ABC-stacked trilayer graphene. ACS Nano 5, 8760-8768 (2011).

58. Dresselhaus, M. S., Jorio, A. \& Saito, R. Characterizing Graphene, Graphite, and Carbon Nanotubes by Raman Spectroscopy. Annu. Rev. Condens. Matter Phys. 1, 89-108 (2010).

59. Jorio, A. Raman Spectroscopy in Graphene-Based Systems: Prototypes for Nanoscience and Nanometrology. ISRN Nanotechnol. 234216 (2012). doi: $10.5402 / 2012 / 234216$

60. Saito, R., Hofmann, M., Dresselhaus, G., Jorio, A. \& Dresselhaus, M. S. Raman spectroscopy of graphene and carbon nanotubes. Advances in Physics 60, 413-550 (2011).

61. Shallcross, S., Sharma, S., Kandelaki, E. \& Pankratov, O. A. Electronic structure of turbostratic graphene. Phys. Rev. B 81, 165105 (2010). 62. Carozo, V. et al. Raman signature of graphene superlattices. Nano Lett. 11, 4527-4534 (2011).

63. Ni, Z., Wang, Y., Yu, T., You, Y. \& Shen, Z. Reduction of Fermi velocity in folded graphene observed by resonance Raman spectroscopy. Phys. Rev. B 77, 235403 (2008).

64. Poncharal, P., Ayari, A., Michel, T. \& Sauvajol, J.-L. Raman spectra of misoriented bilayer graphene. Phys. Rev. B 78, 113407 (2008).

\section{Acknowledgements}

This research was fully supported by the US Department of Energy, Basic Energy Sciences, Division of Materials Science and Engineering, under Contract no. DE-AC02-98CH10886. Fabrication and characterization of the samples was supported in part by the Center for Functional Nanomaterials, Brookhaven National Laboratory, which is supported by the U.S. Department of Energy, Office of Basic Energy Sciences, under Contract No. DESC0012704. The authors would like to thank Xiao Tong at BNL for technical assistance gathering the Raman data and Robert Keller at NIST for technical assistance and useful discussions of the EBSD data. 


\section{Author Contributions}

J.F.P, J.A.G, L.B. and Y.Z conceived and designed the research; J.A.G., L.B. and J.F.P. carried out the PVD growth; J.F.P., L.W. and K.K. acquired and analyzed the TEM data; J.A.G. and J.F.P. acquired and analyzed the Raman data; J.F.P. acquired and analyzed the EBSD data; J.A.G. and J.F.P. prepared the figures and manuscript; All authors made notable contributions to the manuscript.

\section{Additional Information}

Supplementary information accompanies this paper at http://www.nature.com/srep

Competing financial interests: The authors declare no competing financial interests.

How to cite this article: Garlow, J. A. et al. Large-Area Growth of Turbostratic Graphene on Ni(111) via Physical Vapor Deposition. Sci. Rep. 6, 19804; doi: 10.1038/srep19804 (2016).

(c) (i) This work is licensed under a Creative Commons Attribution 4.0 International License. The images or other third party material in this article are included in the article's Creative Commons license, unless indicated otherwise in the credit line; if the material is not included under the Creative Commons license, users will need to obtain permission from the license holder to reproduce the material. To view a copy of this license, visit http://creativecommons.org/licenses/by/4.0/ 